\title{
Prevalence of Over-the-Counter Cosmeceutical Usage and the Impact of a Health Education Intervention in Female Saudi University Students
}

\author{
Faten AlRadini $\mathbb{D}^{\prime}$ \\ Azza El-Sheikh ${ }^{2}$ \\ Noura Bin Jamaan' \\ Hessah Hushan' \\ Wajd Binhuwaimel' \\ Fatemah Alhedaithy' \\ Soad Alanzi \\ 'Department of Clinical Sciences, College \\ of Medicine, Princess Nourah bint \\ Abdulrahman University, Riyadh, II67I, \\ Saudi Arabia; ${ }^{2}$ Department of Basic \\ Health Sciences, College of Medicine, \\ Princess Nourah bint Abdulrahman \\ University, Riyadh, II67I, Saudi Arabia
}

Background: The reckless use of over-the-counter (OTC) cosmeceuticals among females is alarming due to possible health hazards, ranging from allergy to fatal anaphylaxis and toxicity.

Methods: In the current study, we investigated the prevalence of cosmeceutical use among female students at Princess Nourah bint Abdulrahman University (PNU) and tested the effect of a pilot health education program in improving their knowledge. A cross-sectional questionnaire-based study was performed with 515 participants.

Results: A significantly higher percent (81\%) of participants used OTC cosmeceuticals than did not use them. The selection of OTC cosmeceuticals was not affected by socioeconomic status, chronic illness, cosmeceutical cost, or possible cosmeceutical side effects. Students from non-health colleges demonstrated poorer knowledge scores for the hazards of cosmeceuticals, which directed our attention to the importance of health education in this field. Therefore, a pilot interventional health education program was conducted with 54 participants to compare their knowledge before and after the intervention. At the end of the program, a higher percentage of participants had improved their knowledge and realized the need to consult a physician before using OTC cosmeceuticals.

Conclusion: The health education program had a significant impact on knowledge concerning the use of cosmeceuticals, and it is recommended that such programs be included in undergraduate extracurricular activity, especially for female students..

Keywords: over-the-counter, cosmeceuticals, side effects, health education

\section{Plain Language Summary}

In last decade, beauty has been a primary interest of most women in the world, particularly with widespread social media advertisement for fashions and cosmetics. Therefore, we conducted this study to explore how common the use of over-the-counter (OTC) cosmetics was among students of a women's university and what factors might significantly influence this practice. Five hundred and fifteen students were recruited for the study. The results showed that more than $80 \%$ of the students had used OTC cosmetics previously, with a higher percentage of students of non-health care colleges using such cosmetics. This reflects the importance of knowledge in raising awareness of OTC cosmetical hazards. We conducted a health education program and investigated its effect on the students' knowledge and awareness. It resulted in significant improvement in students' awareness of the hazards of OTC cosmetics. Thus, we recommend health education extracurricular activities for students, especially female students, to improve their knowledge and attitude regarding OTC cosmetics and to in turn raise community awareness.
Correspondence: Faten AIRadini

Department of Clinical Sciences, College

of Medicine, Princess Nourah bint

Abdulrahman University, Riyadh, Saudi

Arabia

Email faalradini@pnu.edu.sa 


\section{Introduction}

The irrational use of over-the-counter (OTC) drugs has been considered a major health hazard worldwide, especially with the universal increase in the production and affordability of these drugs. ${ }^{1}$ OTC medications have been reported to be even more consumed than prescribed medications, ${ }^{2,3}$ probably because OTC medications are relatively easier to obtain than prescribed medications, as well as due to the inadequacy of affordable health services and the relatively low cost of OTC medications compared with that of prescribed drugs. ${ }^{3,4}$ A project conducted in Brazil suggested that the determinant factors for selfmedication were mainly influenced by pharmacists' attitudes, family/friends' advice, and uneasiness in contacting healthcare providers. ${ }^{5}$ Unfortunately, prior studies have reported that self-medication with OTC medications is usually done inappropriately and results in hazardous consequences due to administration of higher than the recommended dose, with subsequent iatrogenic adverse effects. ${ }^{6}$

Cosmeceutical OTC medications, which are intermediate products between prescription drugs and cosmetics, create a practical gap in directing public attention to their rational use and potential hazards. ${ }^{7}$ These hazards may range from stinging or burning sensation due to skin irritation to severe allergic contact dermatitis. In addition to affecting consumer health, cosmeceutical OTC drugs also have a significant financial burden on the economics of healthcare, represented by the high cost of treating their possible side effects. ${ }^{8,9}$ It appears that the prevalence of cosmeceutical product usage has been enormously expanding at an unprecedented rate, ${ }^{10}$ especially for topical cosmeceuticals. ${ }^{11}$ However, there is lack of studies that report the exact percentages of cosmeceutical use in individuals with different social, educational and national backgrounds. Nevertheless, as an indicator of such popularity, the global cosmeceutical industry is a rapidly growing market that is expected to reach more than USD 68 billion by 2022 . Increasingly, cost-effective noninvasive means of improving personal appearance of skin and hair are searched for, especially by females and less frequently by men ${ }^{12}$ and even children. ${ }^{13}$ Currently, prescribed medications and medical procedures are being rejected and replaced by OTC medications as cosmeceuticals. ${ }^{8}$ Several types of systemic and topical cosmeceutical treatments are now available to manage conditions such as hyperpigmentation, wrinkles, acne, alopecia and hair damage.
Several studies have been conducted to assess the prevalence of the consumption of OTC products in general, ${ }^{9,14,15}$ but few studies have addressed only OTC agents self-administered for cosmetic reasons. ${ }^{16}$ The latter study reported that most of the sampled population depended on previous experiences choosing OTC drugs and that OTC drugs were preferred over physicianprescribed drugs. The same study proposed the need for institutional extracurricular awareness settings for improving the knowledge of students about the hazards of the irrational use of OTC medications.

In Saudi Arabia, to date, there are no published data on the use of supplemental self-medications, such as oral and/ or topical OTC drugs, for the cosmetic improvement of hair, skin or nails. A single study conducted in Saudi Arabia presented data concerning OTC cosmeceuticals only used in the treatment of acne. ${ }^{2}$ The aim of the current study was to determine the prevalence of OTC cosmeceutical self-medication among female students at Princess Nourah bint Abdulrahman University (PNU) in Riyadh, Saudi Arabia. It also aimed to compare the level of knowledge regarding the use of OTC cosmeceuticals based on different students' sociodemographic characteristics and educational backgrounds. In addition, this study aimed to assess the effect of health education programs in improving students' knowledge. The results of the current study represent a beneficial step toward increasing students' knowledge about different OTC medications available for cosmetic purposes, as well as promoting the rational use of cosmeceuticals.

\section{Materials and Methods Cross-Sectional Questionnaire-Based Study Design}

A cross-sectional questionnaire-based study was performed at PNU to evaluate knowledge regarding using OTC cosmeceuticals among students from both health and non-health colleges. The questionnaire was designed according to data retrieved from a scientific literature search of similar questionnaires performed on the use of OTC products other than cosmeceuticals. ${ }^{2,6,9}$ In addition to demographic questions, the questionnaire included questions about the reasons for OTC administration, factors affecting students' choice of OTC cosmeceuticals, their source of knowledge, the frequency of usage, and their source of purchase. The questionnaire also contained additional questions to assess the level of the students' knowledge about OTC drug indications, 
adverse effects and necessary investigations needed before their use. The main inclusion criterion was being an undergraduate female student at PNU.

\section{Questionnaire Validation, Sample Calculation and Data Collection}

Before data collection, the questionnaire, originally designed in English, was translated to Arabic and then backwards translated to English again for language validation. The questionnaire was validated by five different experts who revised both the Arabic and English versions of the questionnaire. A pilot study was performed with 20 participants, and a few questions were altered in compliance with the participants' and/or experts' suggestions.

The sample size was calculated by using GPower software version 3.1.9.2 (GPower, 2014); with 75\% assumed prevalence, an accepted difference as low as 0.05 , a level of confidence of $95 \%$ (alpha=0.05), and a power of the study of $80 \%$ (beta $=20 \%$ ), the minimal sample size needed was 489 . A total of 540 questionnaires were distributed by 5 undergraduate subinvestigators. The distribution of the questionnaires was performed during a 3-week period in the 2018-2019 academic year, and the completion time for each questionnaire was approximately $10-15$ minutes. The number of respondents to the 540 questionnaires originally distributed was 515, and thus, the response rate fulfilled the sample size.

\section{Pilot Interventional Educational Program}

A follow-up questionnaire-based "before and after" interventional pilot study was performed, which included 54 participants who attended the OTC cosmeceutical awareness program publicly held at PNU. The participants were interviewed before and after the session using pre- and postawareness program questionnaires that measured the prevalence of use and awareness of hazards of OTC cosmeceuticals. The questionnaires included questions about whether the students used OTC products for cosmetics reasons, the rationale for their choices and their sources of knowledge. Additionally, more in-depth questions were asked about specific widespread OTC cosmeceuticals, such as cosmeceuticals containing retinol, doxycycline and minoxidil.

\section{Ethical Consideration}

This work was conducted after institutional review board (IRB) approval from PNU, registered under the number H-01-R-059 on 23/11/2017. A cover consent form and a participant information sheet were distributed, and verbal consent to participate was provided by each participant. The verbal informed consent was approved by the IRB of Princess Nourah bint Abdulrahman University, and the study was conducted in accordance with the Declaration of Helsinki.

\section{Statistical Analysis}

The data are presented as percentage comparative values. The data were analyzed by using IBM SPSS Statistics V21.0. (2014) to descriptively analyze the data and to estimate the internal consistency of the questionnaire, using Cronbach's alpha coefficient, where a value more than 0.70 signified a high level of internal consistency. In addition, knowledge was evaluated by computing the variables related. For categorical variables, Pearson's chi square and Fisher's exact tests were carried out. Differences were considered statistically significant when the $\mathrm{P}$ value was less than 0.05 .

\section{Results}

\section{Sociodemographic Characteristics}

After validation of the questionnaire, the internal consistency of the questionnaire, tested by the SPSS reliability command, was found to be high (Cronbach's alpha of 0.89). Out of the 540 participants, 515 had successfully completed the questionnaire, for a response rate of $95.4 \%$. Five hundred and four $(97.9 \%)$ of the participants were Saudi, and only $11(2.1 \%)$ were non-Saudi. Of all participants, 340 (66\%) were students of non-health colleges, while 175 (34\%) were students of health colleges (medical, pharmacy, dental, nursing and rehabilitation). Most of the participants' ages ranged from 18 to 24 years $(97 \%)$, with only $1 \%$ below 18 and $2 \%$ above 24 years. Concerning the college year of the participants, 98 $(19 \%)$ were in their foundation year, $109(21.2 \%)$ were in their first year, $90(17.5 \%)$ were in their second year, 105 $(20.4 \%)$ were in their third year, $69(13.4 \%)$ were in their fourth year, and $44(8.5 \%)$ were in their fifth year. Concerning the socioeconomic status of the participants, $337(65 \%)$ of them had a family monthly income of 20,000 SR or less, and 178 (35\%) had an income above 20,000 SR. Most participants were single (469; 91.1\%), with only 39 (7.6\%) married and 7 (1.4\%) divorced, out of whom 6 were pregnant, 13 had 1 child, 2 had 2 children, and 1 had 3 or more children. The main sociodemographic parameters of the respondents are listed in Table 1 . Concerning history of chronic illnesses (Table 2), the majority of participants (412; 
Table I Sociodemographic Characteristics of the 515 Sampled PNU Female Students

\begin{tabular}{|c|c|c|}
\hline Variable & Frequency & (Percent) \\
\hline \multicolumn{3}{|l|}{ Nationality } \\
\hline Saudi & 504 & (97.9\%) \\
\hline Non-Saudi & 11 & $(2.1 \%)$ \\
\hline Non-health colleges & 340 & $(66.0 \%)$ \\
\hline Health colleges & 175 & $(34.0 \%)$ \\
\hline Medical & 80 & (15.5\%) \\
\hline Dental & 10 & $(1.9 \%)$ \\
\hline Pharmacy & 14 & $(2.7 \%)$ \\
\hline Rehabilitation & 34 & $(6.6 \%)$ \\
\hline Nursing & 37 & $(7.2 \%)$ \\
\hline \multicolumn{3}{|l|}{ College year } \\
\hline Foundation & 98 & $(19.0 \%)$ \\
\hline First & 109 & $(21.2 \%)$ \\
\hline Second & 90 & (17.5\%) \\
\hline Third & 105 & (20.4\%) \\
\hline Fourth & 69 & (13.4\%) \\
\hline Fifth & 44 & $(8.5 \%)$ \\
\hline \multicolumn{3}{|l|}{ Monthly family income } \\
\hline$<5000$ & 55 & $(10.7 \%)$ \\
\hline $5000-10,000$ & 140 & $(27.2 \%)$ \\
\hline $10,00 \mid-20,000$ & 142 & $(27.6 \%)$ \\
\hline $20,001-30,000$ & 97 & $(18.8 \%)$ \\
\hline $30,001-60,000$ & 53 & $(10.3 \%)$ \\
\hline$>60,000$ & 28 & $(5.4 \%)$ \\
\hline \multicolumn{3}{|l|}{ Social status } \\
\hline Single & 469 & (91.1\%) \\
\hline Married & 39 & $(7.6 \%)$ \\
\hline Divorced & 7 & (I.4\%) \\
\hline \multicolumn{3}{|l|}{ Number of children } \\
\hline $\mathrm{N} / \mathrm{A}^{*}$ & 468 & (90.9\%) \\
\hline None ${ }^{* *}$ & 25 & $(4.9 \%)$ \\
\hline Pregnant & 6 & $(1.2 \%)$ \\
\hline One child & 13 & $(2.5 \%)$ \\
\hline Two children & 2 & $(0.4 \%)$ \\
\hline 3 or more & I & $(0.2 \%)$ \\
\hline
\end{tabular}

Notes: *N/A, not applicable, as the student is single; **None, married with no children.

$80 \%)$ reported having none, while $103(20 \%)$ of the participants had chronic diseases; of these participants, $10(1.9 \%)$ were diabetic, $4(0.8 \%)$ were hypertensive, $22(4.3 \%)$ were asthmatic, 11 (2.1\%) had thyroid disorders, $36(7 \%)$ were anemic, 10 (1.9\%) had depression, and 10 (1.9\%) had other chronic illnesses.

\section{Prevalence of Self-Medication Using OTC} Cosmeceuticals

Out of 515 participants, most $(419 ; 81.4 \%)$ reported using OTC products as cosmeceuticals, while only $96(18.6 \%)$
Table 2 History of Chronic Illness Among the 5 I 5 Sampled PNU Female Students

\begin{tabular}{|l|c|c|}
\hline Chronic Illness & Frequency & (Percent) \\
\hline None & 412 & $(80.0 \%)$ \\
Diabetes mellitus & 10 & $(1.9 \%)$ \\
Hypertension & 4 & $(0.8 \%)$ \\
Bronchial asthma & 22 & $(4.3 \%)$ \\
Thyroid disorders & 11 & $(2.1 \%)$ \\
Anemia & 36 & $(7.0 \%)$ \\
Depression & 10 & $(1.9 \%)$ \\
Other illness & 10 & $(1.9 \%)$ \\
\hline
\end{tabular}

stated that they were nonusers. The prevalence of the use of OTC cosmeceuticals for the hair, skin, and nails and teeth is summarized in Tables 3-5, respectively. Of the participants using OTC cosmeceuticals, nearly half used medications for the treatment of dry skin, and one-third used medications for the treatment of alopecia. The prevalence of OTC cosmeceutical use was significantly higher than that of nonuse in students in all years of college (Table 6). The total number of users of OTC cosmeceuticals was significantly higher than the number of nonusers regardless of specialty. Comparing students from health and non-health colleges concerning the use of the cosmetic OTC showed no significant difference (Figure 1).

Comparing the use of OTC medications among participants with different socioeconomic statuses showed no significant difference in OTC use between low-income $(82 \%)$ and high-income (80\%) students. Similarly, history of chronic illness did not significantly affect the use of OTC products for cosmetic purposes, with the percentage of participants with chronic illnesses who used OTC for

Table 3 Use of Different OTC Cosmeceuticals for the Hair Among the Sampled PNU Female Students

\begin{tabular}{|l|c|c|}
\hline Hair OTC Cosmeceuticals & Frequency* & (Percent) \\
\hline Oral OTC for hair strengthening & 98 & $(19.0 \%)$ \\
Topical OTC for treatment of alopecia & 163 & $(31.7 \%)$ \\
Topical OTC for treatment of thin hair & 60 & $(11.7 \%)$ \\
Topical OTC for strengthening hair & 107 & $(20.8 \%)$ \\
Topical OTC for straightening hair & 99 & $(19.2 \%)$ \\
Topical OTC for curling hair & 61 & $(11.8 \%)$ \\
Topical OTC for treatment of hair dandruff & 95 & $(18.4 \%)$ \\
Topical OTC for treatment of white hair & 9 & $(1.7 \%)$ \\
Topical OTC for treatment of hair lice & 27 & $(5.2 \%)$ \\
Topical OTC for eye lashes & 65 & $(12.6 \%)$ \\
\hline
\end{tabular}

Notes: *Frequency (\%) out of the 515 sampled students; 419 (81.4\%) reported using OTC cosmeceuticals, and 96 (18.6\%) out of the total sampled students reported that they were nonusers. 
Table 4 Use of Different OTC Cosmeceuticals for the Skin Among the Sampled PNU Female Students

\begin{tabular}{|l|c|c|}
\hline Skin OTC Cosmeceuticals & Frequency* & (Percent) \\
\hline Oral OTC for general skin health & 47 & $(9.1 \%)$ \\
Oral OTC for treatment of acne & 44 & $(8.5 \%)$ \\
Topical OTC for treatment of acne & 179 & $(34.8 \%)$ \\
Topical OTC for treatment of acne marks & 113 & $(21.9 \%)$ \\
Topical skin cleansing OTC & 238 & $(46.2 \%)$ \\
Topical OTC for treatment of dry skin & 260 & $(50.5 \%)$ \\
OTC for dry heels & 33 & $(6.4 \%)$ \\
Topical OTC for treatment of vitiligo & 2 & $(0.4 \%)$ \\
Topical OTC for skin tanning & 41 & $(8.0 \%)$ \\
Topical OTC for skin whitening & 63 & $(12.2 \%)$ \\
Topical OTC for treatment of eczema & 41 & $(8.0 \%)$ \\
Topical OTC for treatment of stretch marks & 38 & $(7.4 \%)$ \\
Topical OTC for treatment of bad skin odor & 117 & $(22.7 \%)$ \\
\hline
\end{tabular}

Notes: *Frequency (\%) out of the 515 sampled students; 419 (81.4\%) reported using OTC cosmeceuticals, and $96(18.6 \%)$ out of the total sampled students reported that they were nonusers.

Table 5 Use of Different OTC Products for the Nails and Mouth as Well as Oral Multivitamin Medications Among the Sampled PNU Female Students

\begin{tabular}{|l|c|c|}
\hline OTC Cosmeceuticals & Frequency* & (Percent) \\
\hline Topical OTC for general nail health & 108 & $(21.0 \%)$ \\
Topical OTC for fungal nail infections & 16 & $(3.1 \%)$ \\
Topical OTC for broken nails & 81 & $(15.7 \%)$ \\
OTC for cleansing the mouth & 166 & $(32.2 \%)$ \\
OTC for teeth whitening & 169 & $(32.8 \%)$ \\
OTC for treatment of bad mouth odor & 90 & $(17.5 \%)$ \\
Oral OTC multivitamins & 163 & $(31.7 \%)$ \\
\hline
\end{tabular}

Notes: *Frequency (\%) out of the 515 sampled students; 419 (81.4\%) reported using OTC cosmeceuticals, and 96 (18.6\%) out of the total sampled students reported that they were nonusers.

cosmetic purposes $(80 \%)$ being identical to that of participants who did not suffer from chronic illnesses $(80 \%)$.

The frequency of use of OTC cosmeceuticals among the participants varied from once daily to once yearly (Figure 2A). The factors affecting their choice of OTC cosmeceutical products before purchase mainly included the indication of use, the product brand, presence of widely known side effects and convenience of dosage form (Figure 2B). There was a significantly higher percentage of participants who declared that the price was not a crucial factor affecting their choice of OTC medication, whether participants were from health $(66 \%)$ or non-health (76\%) colleges. Similarly, significantly higher percentages of participants from both health $(63 \%)$ and non-health $(70 \%)$ colleges reported that the presence of side effects also did not affect the choice of OTC cosmeceutical.
A significantly higher percentage of participants purchased their OTC cosmeceuticals from a pharmacy $(70 \%$ and $73 \%$ ) than from the internet $(16 \%$ and $17 \%)$ or a supermarket (15\% and $13 \%)$, with no significant differences between health and non-health college participants, respectively. A comparison between health and non-health college students showed that both significantly preferred to purchase OTC cosmeceuticals from pharmacies $(73 \%$ and $70 \%$, respectively), with no significant difference between them (Pearson chi-square test $\mathrm{P}$ value of $0.015 ;<0.05$ ).

\section{Knowledge and Awareness Assessment}

The majority of the participants in the cross-sectional study reported that their source of information governing their choice of OTC drug was pharmacist advice (44\%), in addition advice from family members and friends, selfacquired information, recommendations from celebrities, and advertisements on the TV and internet (Figure 2C). Concerning side effects, among the users of OTC cosmeceuticals, only $24 \%$ were advised by a doctor/pharmacist about the side effects of cosmeceuticals, while the majority relied on the media, internet, family and friends. A significantly higher percentage of participants (66\%) believed that self-medication using cosmeceuticals was acceptable Similarly, a significantly higher percentage of participants believed that OTCs of natural origins as plants were safer $(63 \%)$ than synthetic $(2 \%)$ and semisynthetic (5\%) OTC drugs, while $30 \%$ of participants believed that there was no completely safe source of OTC cosmeceuticals. A significantly higher percentage of participants $(61 \%)$ believed that topical OTC drugs were less hazardous than injected (4\%) and oral (4\%) OTC drugs, while $31 \%$ believed that OTC cosmeceuticals were dangerous regardless of the route of administration. A significantly higher percentage of OTC users did not suffer from side effects $(76 \%)$, while the minority suffered from gastrointestinal symptoms such as nausea, vomiting and diarrhea or skin and hair problems such as rash, itching, change in skin color or alopecia. Nevertheless, $77 \%$ of participants thought that it is advisable to perform laboratory investigations before the use of any OTC, even for cosmetic purposes.

To assess the knowledge of the participants, a knowledge score was computed by collectively taking the average of the answers of 4 knowledge-related questions into one variable and categorizing the score as poor ( $0-1$ correct answers out of 4$)$, moderate (2 correct answers out of 4 ) and excellent (3-4 correct answers out 
Table 6 Comparison Between OTC Cosmeceutical Users and Nonusers in Different College Years

\begin{tabular}{|c|c|c|c|c|c|c|c|c|}
\hline \multicolumn{2}{|c|}{ Previous Use of OTCs } & \multicolumn{6}{|c|}{ College Year } & \multirow[t]{2}{*}{ Total } \\
\hline & & Foundation & First & Second & Third & Fourth & Fifth & \\
\hline No & $\begin{array}{c}\text { Count } \\
\text { \% Previously use of OTC } \\
\text { Std. Residual }\end{array}$ & $\begin{array}{c}18 \\
18.8 \% \\
-0.1\end{array}$ & $\begin{array}{c}24 \\
25.0 \% \\
0.8\end{array}$ & $\begin{array}{l}1 \mathrm{I} \\
\mathrm{II} .5 \% \\
-1.4\end{array}$ & $\begin{array}{c}26 \\
27.1 \% \\
1.5\end{array}$ & $\begin{array}{c}16 \\
16.7 \% \\
0.9\end{array}$ & $\begin{array}{c}1 \\
1.0 \% \\
-2.5\end{array}$ & $\begin{array}{c}96 \\
100.0 \%\end{array}$ \\
\hline Yes & $\begin{array}{c}\text { Count } \\
\text { \% Previously use of OTC } \\
\text { Std. Residual }\end{array}$ & $\begin{array}{c}80 \\
19.1 \% \\
0.0\end{array}$ & $\begin{array}{c}85 \\
20.3 \% \\
-0.4\end{array}$ & $\begin{array}{c}79 \\
18.9 \% \\
0.7\end{array}$ & $\begin{array}{c}79 \\
18.9 \% \\
-0.7\end{array}$ & $\begin{array}{c}53 \\
12.6 \% \\
-0.4\end{array}$ & $\begin{array}{c}43 \\
10.3 \% \\
1.2\end{array}$ & $\begin{array}{c}419 * \\
100.0 \%\end{array}$ \\
\hline Total & $\begin{array}{c}\text { Count } \\
\% \text { Previously use of OTC }\end{array}$ & $\begin{array}{c}98 \\
19.0 \%\end{array}$ & $\begin{array}{c}109 \\
21.2 \%\end{array}$ & $\begin{array}{c}90 \\
17.5 \%\end{array}$ & $\begin{array}{c}105 \\
20.4 \%\end{array}$ & $\begin{array}{c}69 \\
13.4 \%\end{array}$ & $\begin{array}{c}44 \\
8.5 \%\end{array}$ & $\begin{array}{c}515 \\
100.0 \%\end{array}$ \\
\hline
\end{tabular}

Note: *Pearson's chi square test showed a P value of $0.012(<0.05)$.

of 4) knowledge. Our results showed that overall, the significantly more health and non-health college participants had poor knowledge scores (47\%) than moderate $(28 \%)$ or excellent $(25 \%)$ scores. A detailed comparison between participants from health and non-health colleges is shown (Figure 3).

\section{Intervention to Assess Knowledge Pre- and Post-Awareness Program}

A total of 54 participants were interviewed before and after the interventional awareness program, which was publicly held at PNU as an interactive awareness program to enhance knowledge of the use of OTC cosmetics. Approximately $25 \%$ of participants realized that there was no dosage form of OTC cosmeceuticals that could be considered safe and that these drugs might be hazardous regardless of drug form. After the awareness program, the percentage of participants who realized that there was no dosage form of OTC cosmeceuticals considered safe increased to $91 \%$ (Figure 4). In addition, on examining the knowledge of participants about

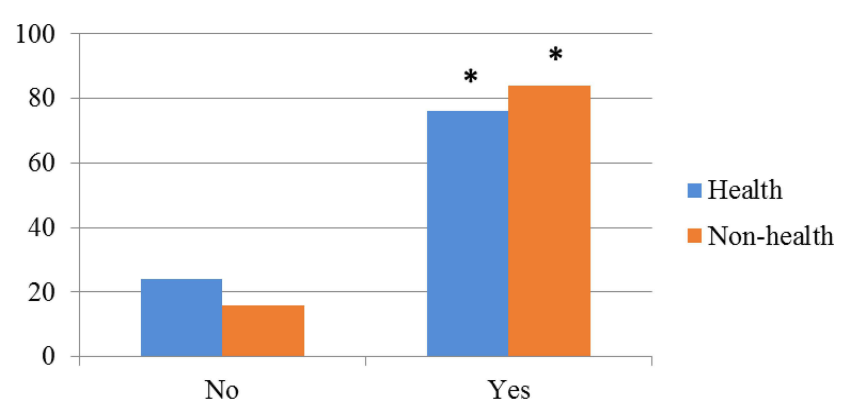

Figure I Comparison between health (blue bar) and non-health (Orange bar) college students concerning the use (yes) or nonuse (no) of OTC cosmeceuticals. Notes: Data represent the calculated comparative percentage from a total of 515 samples. *Significant difference with $\mathrm{P}<0.05$. the side effects of three medications used as OTC cosmeceutical, namely, retinol, doxycycline and minoxidil, 30\%, 91\%, and $84 \%$, respectively, did not try to answer and admitted that they did not know the correct answer before the awareness program, while after the awareness program, $88 \%, 86 \%$, and $83 \%$, respectively, responded with the correct answer about the side effects of these 3 drugs. In another question about who to consult before using OTC medications as cosmeceuticals, before the program, only $7 \%$ of participants responded that a doctor should be consulted, while the rest indicated that they would consult the pharmacist, read the leaflet, ask family members or friends or check the internet in the preawareness program assessment (Figure 5A). On the other hand, after the awareness program, on answering the same question, the percentage of participants who would choose to consult a doctor increased to $96 \%$ (Figure 5B).

\section{Discussion}

Cosmeceuticals are compounds with active ingredients that have beneficial physiological effects due to their pharmacological actions compared with the lack of such actions in inert cosmetics. In the current study, significantly higher levels of users than nonusers of OTC cosmeceuticals were encountered among female students at PNU. This higher level of consumption of these drugs in the present study was independent of the socioeconomic status and academic year of participants, as well as the price of the OTC product, its brand, or the presence of side effects. Previous studies indicated that the cause of the significant use of OTC cosmeceuticals was most likely because of a lack of insurance coverage for prescription products used as cosmeceuticals, the inconvenience of having to go to a physician to obtain prescription medications, and the amazing unrealistic claims advertised 


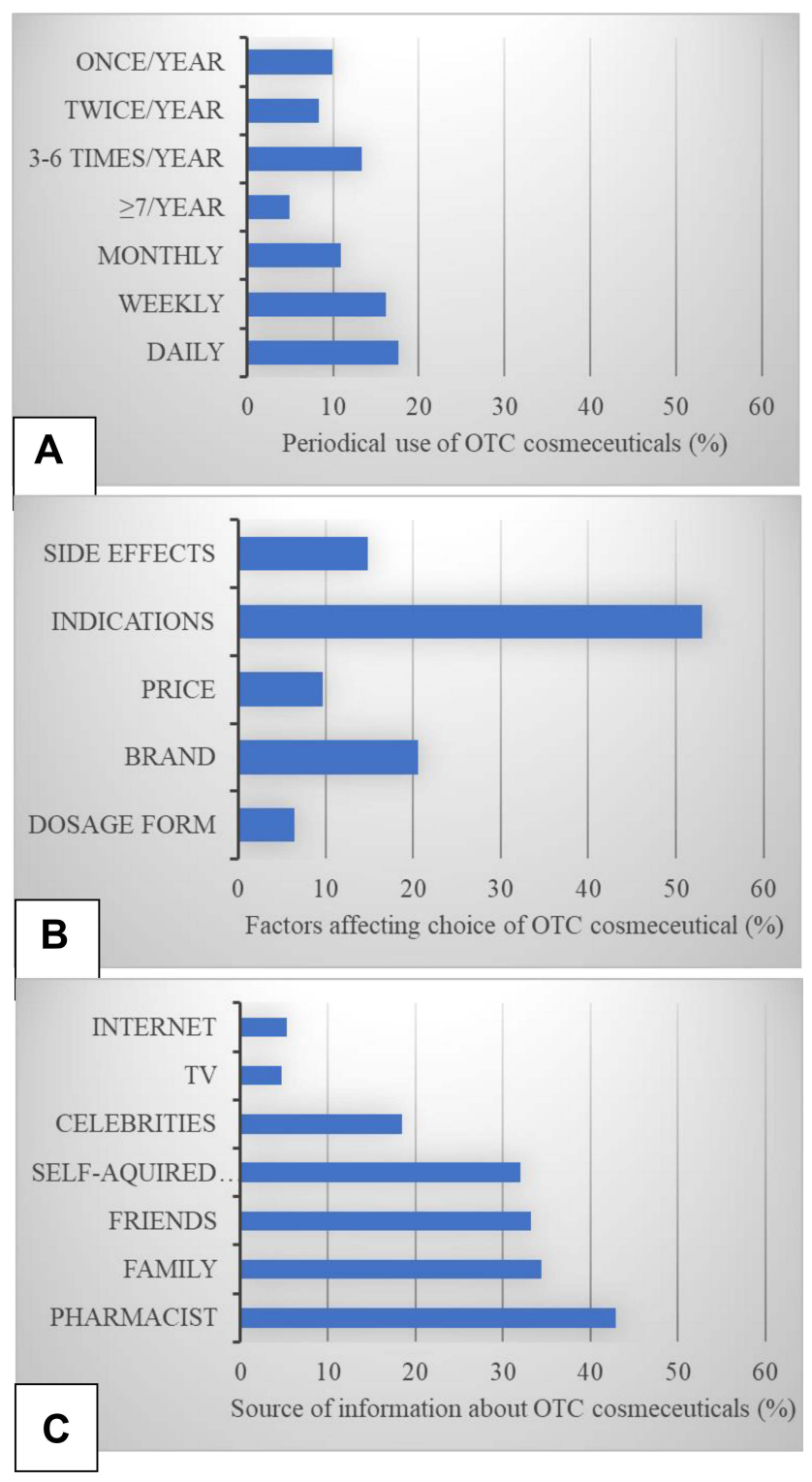

Figure 2 Percentage using OTC cosmeceuticals (A), the factors affecting the choice of purchasers (B) and the source of information before purchase (C).

by some OTC skin and hair care products. ${ }^{17}$ The percentage of regular daily users of OTC products for cosmeceutical reasons in this study, however, was less than anticipated (91; $17.7 \%)$. This is probably because the current study limited the age groups to those of university students, with most participants being from the same ethnic background, and did not include more diverse groups and older age groups who would need to apply OTC cosmeceuticals more frequently. ${ }^{18}$

In the present study, a significant number of participants thought that the topical application of OTC cosmeceuticals would pose no hazards. This idea was not changed until after they completed the interventional awareness program, with the post-awareness program assessment showing that the majority of participants realized that there was no medication form without hazards, including topical OTC cosmeceuticals. A study previously reported that the users of OTC cosmeceuticals might have different potentially false perceptions of the use of such medications and that they would possibly think that, since they are applied topically, such compounds do not have any systemic side effects. ${ }^{17}$ The current study focused only on pharmacy-sold OTC cosmeceuticals and did not address homemade chemical or herbal remedies, although these ethnomedicines were long widely used by rural inhabitants, herbalists, traditional healers and even beauty salon hairdressers. ${ }^{19}$ It is publicly accepted that phytocompounds of natural plant origins might be safer than synthetic compounds, which was also the case in the current study, in which the majority of participants believed that natural sources were safer than synthetic sources, a belief that was corrected after attending the awareness program. Notably, using new techniques such as nanotechnology to manipulate cosmeceuticals at an atomic or molecular level might cause an increase in the bioavailability of active ingredients of cosmeceutical products with the prolongation of their effects, resulting in the penetration of nanoparticles through the skin, potentially causing systemic hazardous side effects. ${ }^{20}$

The lack of awareness of potential hazards of cosmeceuticals might cause severe and even fatal adverse effects. One prominent example of such hazards is the use of retinoic acid, the most important active metabolite of vitamin $\mathrm{A}$, as a skincare cosmeceutical, which might cause congenital teratogenicity in pregnant women, among other side effects. ${ }^{21,22}$ As derivatives of natural compounds and topically applied, vitamin A and retinoic acid may deceive many patients into using them without realizing their hazards. In the current study, the majority of participants did not know the side effects of OTC cosmeceuticals containing retinoic acid, which was significantly reversed after attending the awareness program. Another example is the topical use of the well-known vasodilator minoxidil for the treatment of hair loss, which might cause, among other drastic side effects, allergic contact dermatitis of the scalp and progressive hair loss, especially when its administration is abruptly stopped. ${ }^{23}$ Doxycycline is a third example of a topical compound widely used as an OTC cosmeceutical for the treatment of acne vulgaris. Even with topical application, doxycycline might cause photosensitivity to the skin and 


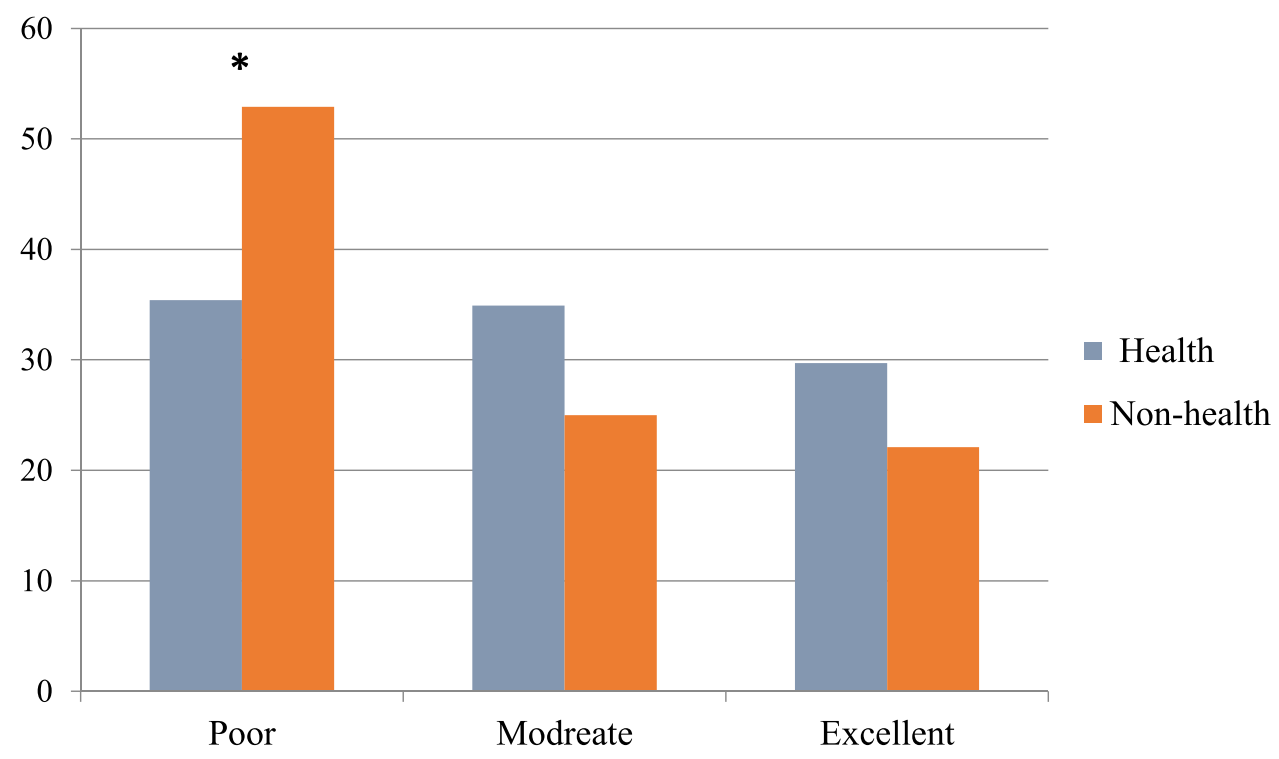

Figure 3 Comparison between health (blue bar) and non-health (Orange bar) college students knowledge scores regarding the use of OTC cosmeceuticals. Notes: Data represent the calculated comparative percentage, where the knowledge score was considered poor (0-I), moderate (2) or excellent (3-4) (out of 4 knowledge questions were answered correctly). *Significant difference with $\mathrm{P}<0.05$.

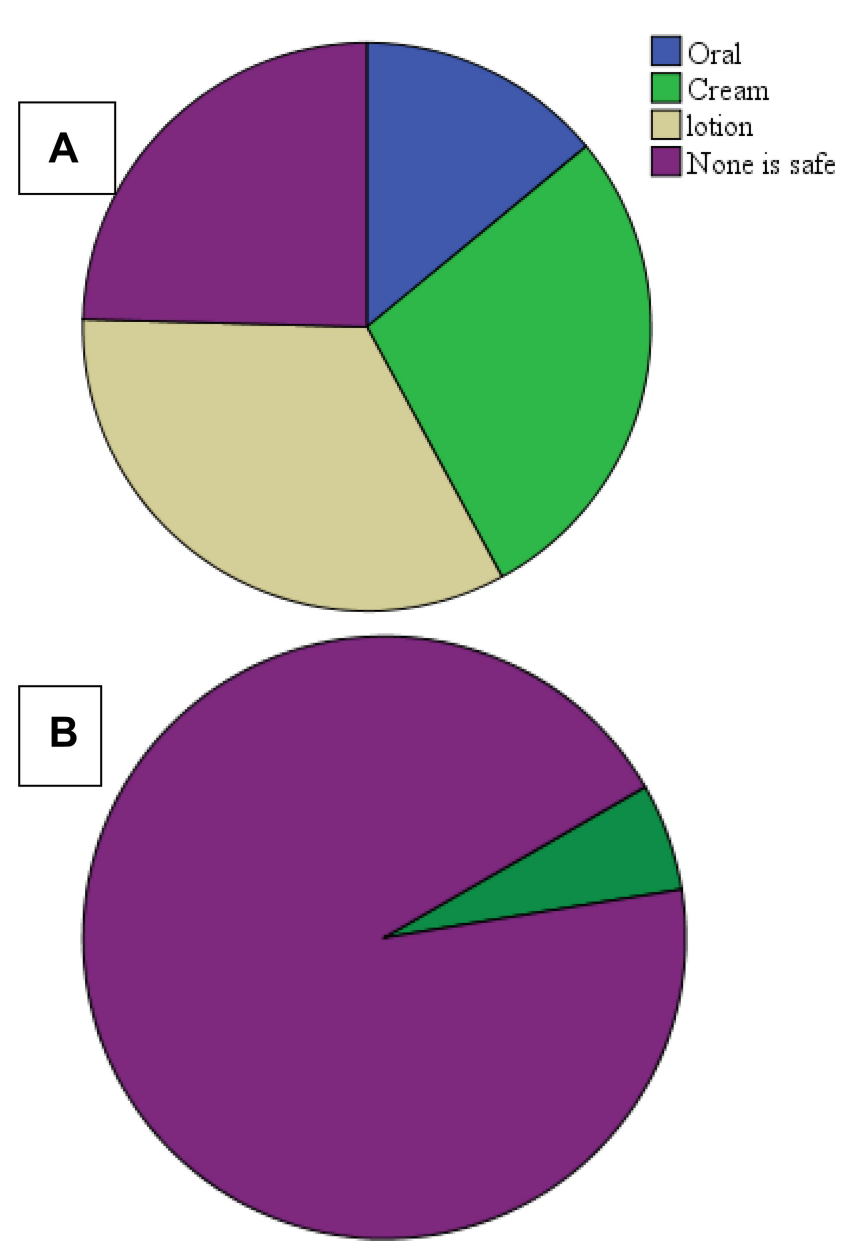

Figure 4 Comparison between before $(\mathbf{A})$ and after $(\mathbf{B})$ the awareness workshop concerning which dosage formulation of OTC cosmeceuticals is considered safe. Data represent the calculated comparative percentage from a total of 54 samples. teratogenic effects, among other hazardous adverse effects. $^{24}$ As in the case of retinoic acid side effects, in the current study, the participants only knew about the hazards of the use of minoxidil and doxycycline after attending the awareness program.

Previous studies implemented awareness programs to increase community knowledge, ${ }^{25}$ especially related to drug side effects. ${ }^{26}$ One of the potential limitations of the current study is that the impact on awareness was assessed immediately after the program was disseminated. However, several studies have relied on such a quick response to evaluate the effect of their awareness programs. $^{27-29}$

It would be beneficial to address such awareness programs to healthcare providers, although they might seem not in need of them. In the current study, there was no significant difference in the use, knowledge and awareness of hazards, as well as other parameters of OTC use as cosmeceuticals, between health and non-health college students. This might be because of the lack of academic curricula with any information dealing with this matter in the health colleges. Continuing medical education was reported to be effective in improving clinician information to overcome patient medication misuse. ${ }^{25,30}$ Directing the attention of future healthcare providers to the hazards of the use of OTC cosmeceuticals would yield tangible results, as they could use this knowledge to efficiently manage their patients and could spread this knowledge to 


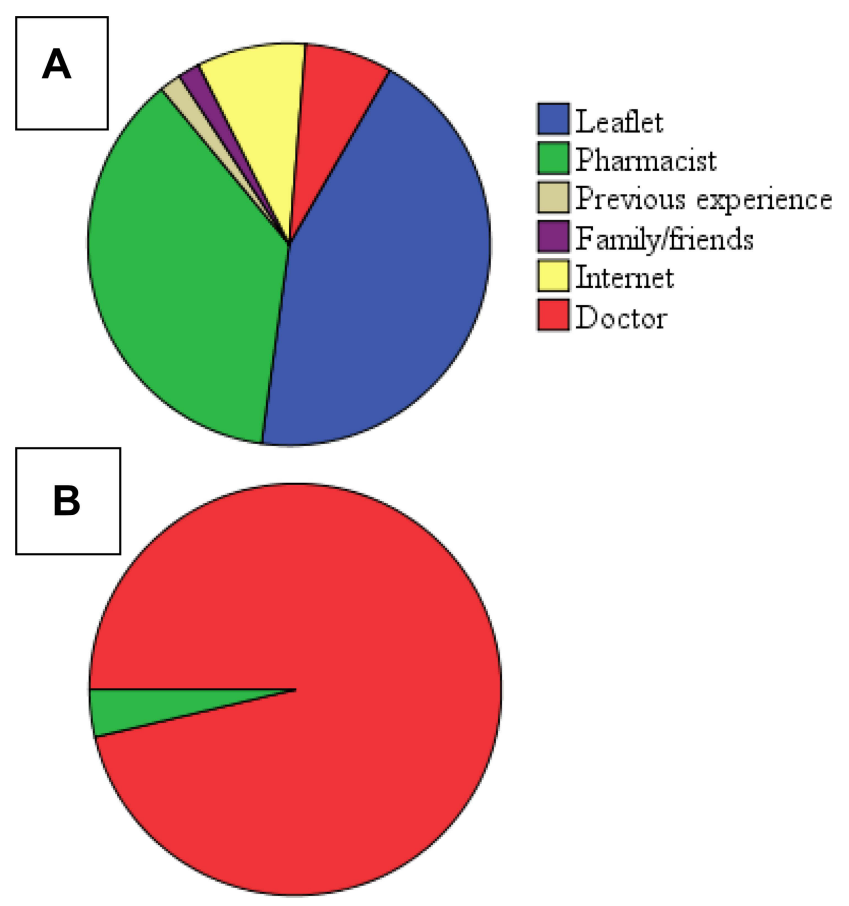

Figure 5 Comparison between before $(\mathbf{A})$ and after $(\mathbf{B})$ the awareness workshop concerning who/what to consult before using OTC cosmeceuticals. Data represent the calculated comparative percentage from a total of 54 samples.

future undergraduates under their supervision. Additional studies are necessary to evaluate the knowledge and awareness of pharmacists, especially those working at community pharmacies, about the adverse effects and drug interactions of OTC cosmeceuticals, as pharmacists are the main source of information for a large public sector.

\section{Conclusions}

We conclude that variable percentages of the PNUsampled population were using OTC drugs for different cosmetic reasons without being fully aware of their proper indications, method of use or possible adverse effects. Cosmeceutical OTC price and side effects, as well as student socioeconomic status, did not influence the students' choices when purchasing OTC cosmeceuticals. However, being a student in a health college influenced the knowledge score for cosmeceutical use, suggesting a role of health education. Performing an interventional educational program had a high impact on the awareness of students and their knowledge about using OTC cosmeceuticals and significantly influenced their awareness about possible consequences of the use of OTC cosmeceuticals.

\section{Abbreviations}

OTC, over-the-counter; PNU, Princess Nourah bint Abdulrahman University.

\section{Data Sharing Statement}

Data are available with the primary author.

\section{Ethics Approval and Informed Consent}

This work was conducted after institutional review board (IRB) approval from PNU, registered under the number H-01-R-059 on 23/11/2017.

\section{Consent for Publication}

A cover consent form and a participant information sheet were distributed, and verbal consent to participate was provided by each participant.

\section{Acknowledgments}

The authors are grateful to Princess Nourah bint Abdulrahman University for supporting this research through Future pioneers' program in Deanship of scientific research. A provisional abstract of this work was accepted for a poster presentation at the Second Medical Students Research Forum at King Saud University, Riyadh, Saudi Arabia (1-2 February 2019).

\section{Author Contributions}

All authors made a significant contribution to the work reported, whether that is in the conception, study design, execution, acquisition of data, analysis and interpretation, or in all these areas; took part in drafting, revising or critically reviewing the article; gave final approval of the version to be published; have agreed on the journal to which the article has been submitted; and agree to be accountable for all aspects of the work.

\section{Funding}

This research project was funded by the Deanship of Scientific Research, Princess Nourah bint Abdulrahman University, through the Pioneer Researcher Funding Program, Grant No 39-PR-2.

\section{Disclosure}

The authors report no conflicts of interest in this work. 


\section{References}

1. World Health Organization. The world medicines situation 2011. Available from: http://www.who.int/medicines/areas/policy/world_ medicines_situation/WMS_ch6_wPricing_v6.pdf. Accessed August 13, 2021.

2. Alshehri MD, Almutairi AT, Alomran AM, Alrashed BA, Kaliyadan F. Over-the-counter and prescription medications for acne: a cross-sectional survey in a sample of university students in Saudi Arabia. Indian Dermatol Online J. 2017;8(2):120-123. doi:10.4103/2229-5178.202273

3. Panda A, Pradhan S, Mohapatro G, Kshatri JS. Predictors of over-the-counter medication: a cross-sectional Indian study. Perspect Clin Res. 2017;8(2):79-84. doi:10.4103/2229-3485.203043

4. Decker A, Graber EM. Over-the-counter acne treatments: a review. $J$ Clin Aesthet Dermatol. 2012;5:32-40.

5. Filho AI, Lima-Costa MF, Uchôa E. Bambuí project: a qualitative approach to self-medication. Cad Saúde Pública. 2004;20 (6):1661-1669. doi:10.1590/S0102-311X2004000600025

6. Tesfamariam S, Anand IS, Kaleab G, et al. Self-medication with over the counter drugs, prevalence of risky practice and its associated factors in pharmacy outlets of Asmara, Eritrea. BMC Public Health. 2019;19(1):159. doi:10.1186/s12889-019-6470-5

7. Barros BS, Zaenglein AL. The use of cosmeceuticals in acne: help or hoax? Am J Clin Dermatol. 2017;18(2):159-163. doi:10.1007/ s40257-016-0249-6

8. Bowe WP, Shalita AR. Effective over-the-counter acne treatments. Semin Cutan Med Surg. 2008;27(3):170-176. doi:10.1016/j. sder.2008.07.004

9. Albusalih FA, Naqvi AA, Ahmad R, Ahmad N. Prevalence of self-medication among students of pharmacy and medicine colleges of a public sector university in Dammam city, Saudi Arabia. Pharmacy (Basel, Switzerland). 2017;5(3):51.

10. Lee M. The expandin "cosmeceutical" conundrum: the growing need for heightened FDA regulations in the face of modern day cosmeceuticals. 2011; Available from: https://dash.harvard.edu/handle/1/ 8789605. Accessed August 13, 2021.

11. Nolan BV, Levender MM, Davis SA, Feneran AN, Feldman SR, Feldman SR. Trends in the use of topical over the counter products in the management of dermatologic disease in the United States. Dermatol Online J. 2012;18(2):1. doi:10.5070/D33PK0X5ZV

12. Draelos ZD. Cosmeceuticals for male skin. Dermatol Clin. 2018;36 (1):17-20. doi:10.1016/j.det.2017.09.003

13. Pham AK, Dinulos JG. Cosmeceuticals for children: should you care? Curr Opin Pediatr. 2014;26(4):446-451. doi:10.1097/ MOP.0000000000000105

14. Barrenberg E, Garbe E. Use of over-the-counter (OTC) drugs and perceptions of OTC drug safety among German adults. Eur J Clin Pharmacol. 2015;71(11):1389-1396. doi:10.1007/s00228-015-1929-5

15. Helal RM, Abou-ElWafa HS. Self-medication in university students from the city of Mansoura, Egypt. J Environ Public Health. 2017;2017:9145193. doi:10.1155/2017/9145193

16. Almalak H, Albluwi AI, Alkhelb DA, et al. Students' attitude toward use of over the counter medicines during exams in Saudi Arabia. Saudi Pharm J. 2014;22(2):107-112. doi:10.1016/j.jsps.2013.02.004
17. Levin J, Momin SB. How much do we really know about our favorite cosmeceutical ingredients? J Clin Aesthet Dermatol. 2010;3 (2):22-41.

18. Messaraa C, Doyle L, Mansfield A, O'Connor C, Mavon A. Ageing profiles of Caucasian and Chinese cohorts - focus on hands skin. Int J Cosmet Sci. 2019;41(1):79-88. doi:10.1111/ics.12514

19. Zaid AN, Jaradat NA, Eid AM, Al Zabadi H, Alkaiyat A, Darwish SA. Ethnopharmacological survey of home remedies used for treatment of hair and scalp and their methods of preparation in the West Bank-Palestine. BMC Complement Altern Med. 2017;17(1):355. doi:10.1186/s12906-017-1858-1

20. Lohani A, Verma A, Joshi H, Yadav N, Karki N. Nanotechnologybased cosmeceuticals. ISRN Dermatol. 2014;2014:843687. doi: $10.1155 / 2014 / 843687$

21. Nijhof S, Kutz P, Schulze R, Schaefer C, Menke T, Roll C. Congenital intermittent third-degree atrioventricular block associated with retinoid exposure in pregnancy. Z Geburtshilfe Neonatol. 2015;219(6):289-292. doi:10.1055/s-0035-1550002

22. Shirazi M, Abbariki E, Pirjani R, Akhavan S, Dastgerdy E. Congenital microtia in a neonate due to maternal isotretinoin exposure 1 month before pregnancy: case report. J Obstet Gynaecol Res. 2015;41(6):975-978. doi:10.1111/jog.12644

23. Rossi A, Cantisani C, Melis L, Iorio A, Scali E, Calvieri S. Minoxidil use in dermatology, side effects and recent patents. Recent Pat Inflamm Allergy Drug Discov. 2012;6(2):130-136. doi:10.2174/ 187221312800166859

24. Goetze S, Hiernickel C, Elsner P. Phototoxicity of doxycycline: a systematic review on clinical manifestations, frequency, cofactors, and prevention. Skin Pharmacol Physiol. 2017;30(2):76-80. doi:10.1159/000458761

25. Finnell JT, Twillman RK, Breslan SA, Schultz J, Miller L. The role of continuing medical education in increasing enrollment in prescription drug monitoring programs. Clin Ther. 2017;39(9):1896-1902.e2. doi:10.1016/j.clinthera.2017.07.040

26. Jha N, Rathore DS, Shankar PR, et al. Effect of an educational intervention on knowledge and attitude regarding pharmacovigilance and consumer pharmacovigilance among community pharmacists in Lalitpur district, Nepal. BMC Res Notes. 2017;10(1):4. doi:10.1186/ s13104-016-2343-5

27. Schnaith AM, Evans EM, Vogt C, et al. An innovative medical school curriculum to address human papillomavirus vaccine hesitancy. Vaccine. 2018;36(26):3830-3835. doi:10.1016/j.vaccine.2018.05.014

28. Mora AS, Madrigal JM, Jordan L, Patel A. Effectiveness of an educational intervention to increase human papillomavirus knowledge in high-risk minority women. J Low Genit Tract Dis. 2018;22 (4):288-294. doi:10.1097/LGT.0000000000000386

29. Alraie NA, Saad AA, Sabry NA, Farid SF. Adverse drug reactions reporting: a questionnaire-based study on Egyptian pharmacists' attitudes following an awareness workshop. J Eval Clin Pract. 2016;22 (3):349-355. doi:10.1111/jep.12484

30. Ganesan S, Sandhiya S, Reddy KC, Subrahmanyam DK, Adithan C. The impact of the educational intervention on knowledge, attitude, and practice of pharmacovigilance toward adverse drug reactions reporting among health-care professionals in a tertiary care hospital in South India. J Nat Sci Biol Med. 2017;8(2):203-209. doi:10.4103/ 0976-9668.210014 


\section{Publish your work in this journal}

Clinical, Cosmetic and Investigational Dermatology is an international, peer-reviewed, open access, online journal that focuses on the latest clinical and experimental research in all aspects of skin disease and cosmetic interventions. This journal is indexed on CAS
The manuscript management system is completely online and includes a very quick and fair peer-review system, which is all easy to use. Visit http://www.dovepress.com/testimonials.php to read real quotes from published authors.

Submit your manuscript here: https://www.dovepress.com/clinical-cosmetic-and-investigational-dermatology-journal 